\title{
Review-Engineering the Selectivity of the DNA-SWCNT Sensor
}

\author{
Justyna Kupis-Rozmysłowicz, = Alessandra Antonucci, $=, *$ and Ardemis A. Boghossian ${ }^{* *, z}$ \\ Institute of Chemical Sciences and Engineering (ISIC) École Polytechnique Fédérale de Lausanne (EPFL), \\ 1015-Lausanne, Switzerland
}

\begin{abstract}
Single-walled carbon nanotubes (SWCNTs) demonstrate a unique combination of optical, chemical, and physical properties that render them suitable for a variety of sensing applications. Their photostable near-infrared (nIR) fluorescence emissions are highly sensitive to perturbations in the surrounding SWCNT environment, enabling optical sensors with single-molecule detection limits. Despite these immanent advantages, SWCNTs lack the inherent molecular recognition capabilities required for selective sensing applications. One approach to tuning sensor selectivity is to engineer synthetic and biological wrappings that cover the nanotube's surface in a manner that limits chemical access to the surface to specific target analytes. Among the numerous possible wrappings, deoxyribonucleic acid (DNA) has emerged as the most studied polymeric wrapping. In addition to the sequence-dependent tunability DNA offers in engineering selectivity, DNA assumes a peculiar helical wrapping conformation along the SWCNT surface that has been the focus of many experimental and computational studies. In this review, we summarize some of the major findings in the field, focusing on the underlying molecular interactions responsible for the conformational and molecular recognition elements of the wrapping. Special focus is given to characterizing the nucleotide binding affinity, DNA sequence dependency, DNA length variation, SWCNT chirality, and sugar backbone (RNA vs. DNA) contributions to the wrapping conformation and SWCNT fluorescence. This article concludes with an assessment of the latest DNA-SWCNT-based sensing platforms used for the selective, single- and multi-modal detection of target analytes.

() 2016 The Electrochemical Society. [DOI: 10.1149/2.0111608jss] All rights reserved.
\end{abstract}

Manuscript submitted March 7, 2016; revised manuscript received April 12, 2016. Published April 29, 2016. This paper is part of the JSS Focus Issue on Nanocarbons in Sensing Applications.

Since their introduction in the early $60 \mathrm{~s},{ }^{1}$ biosensors have become an indispensable part of our daily lives, finding disparate applications in a variety of analytical fields ranging from biomedical diagnostics to environmental monitoring to food technology. ${ }^{2}$ Notably, optical biosensors based on fluorescent-light emission, which exploit diverse fluorescent probes as labeling agents for the recognition units, have emerged as highly sensitive, rapid, reproducible, and simple-to-operate analytical tools capable of quantitatively monitoring specific molecular interactions in real time. ${ }^{3,4}$ However, several drawbacks limit their application in more complex chemical and biological environments. For example, typical fluorescence-based sensors conventionally employ organic fluorophores that emit fluorescence with visible wavelengths that do not penetrate well through biological tissue. ${ }^{5}$ Furthermore, such fluorophores are often subject to rapid photobleaching, thus compromising their sensor lifetimes. These limitations are unfavorable for long-term in vivo sensing ${ }^{6}$ for diagnostic applications. Recent research is thus focused on finding new technologies and materials to engineer novel sensing platforms with increased sensitivity and selectivity over extended periods of time.

Single-walled carbon nanotubes (SWCNTs) have become widely used in a new generation of optical transducers with inherent properties that circumvent many of the aforementioned limitations, making them exceptionally promising materials for biosensor design. ${ }^{7}$ SWCNTs are particularly photostable fluorophores that emit light in the near-infrared (nIR) range without evidence of blinking or photobleaching after prolonged exposure to high intensity excitations. ${ }^{8,9}$ In the nIR light range between 700 and $1100 \mathrm{~nm}$, biological moieties like blood, skin, and organ tissues are optically transparent and do not interfere with SWCNT fluorescence. ${ }^{10}$ In addition, their extremely small size, fiber-like shape, and large surface area allow SWCNTs to interact at cellular and molecular levels (e.g. femtomolar-, ${ }^{11}$ and attomolar-range ${ }^{12}$ ), enabling singlemolecule detection limits that are especially desirable for sensing applications.

\footnotetext{
$=$ These authors contributed equally to this work.

* Electrochemical Society Student Member.

***Electrochemical Society Member.

${ }^{\mathrm{z}}$ E-mail: ardemis.boghossian@epfl.ch
}

Despite providing significant advantages in terms of signal brightness, photostability, multimodality, multicolored-light emission, sensitivity, and tissue transparency, carbon nanotubes need to be engineered for analyte selectivity. The selectivity of SWCNT-based architectures can be tuned by engineering a specific coating that can cover the nanotube's surface, imparting SWCNTs with enhanced recognition properties. Since covalent functionalization diminishes SWCNT fluorescence, a key element in optical sensing, these coatings rely on non-covalent means of immobilization. In the past decade, researchers have exploited the intrinsic specificity of many naturally occurring biomolecules and polymers to enrich SWCNTs with exquisite molecular recognition capabilities. Among the different bioconjugation approaches, non-covalent functionalization with deoxyribonucleic acid (DNA) has been the most extensively applied method for developing novel SWCNT-based molecular recognition systems. ${ }^{13}$ Because of its peculiar structure ${ }^{14-16}$ and pronounced flexibility, DNA possesses many desirable characteristics for the fabrication of stable, highly dispersible, non-toxic nano-complexes with unusual multifunctional capabilities. ${ }^{17}$ Moreover, the possibility of having easy access to an incredibly large molecular library of DNA structures, sequences, and lengths for engineering SWCNT surfaces brings forth the opportunity to develop a new generation of smart optical sensors for the selective detection of a plethora of different analytes. ${ }^{18-20}$

To this end, a fundamental understanding of the key elements influencing the interaction between DNA and carbon nanotubes is very crucial. ${ }^{18}$ DNA structural changes in response to nucleotide composition or different environmental conditions may strongly affect DNA binding and molecular recognition behavior. To date, the major aspects of DNA-SWCNT interactions, which include binding geometry, base sequence, orientation, and changes in polarity, have been systematically investigated in both theoretical and experimental studies. In this review, we will analyze the most important factors that have been shown to significantly influence DNA non-covalent wrapping mechanisms and the resulting selectivity. We will focus our attention on describing the distinct components that may control the molecular recognition elements of DNA-SWCNTs. In doing so, we will illustrate several examples of successfully developed DNASWCNT complexes for the selective detection of target analytes such as dopamine, nitric oxide (NO), DNA strands, and divalent metal ions. 


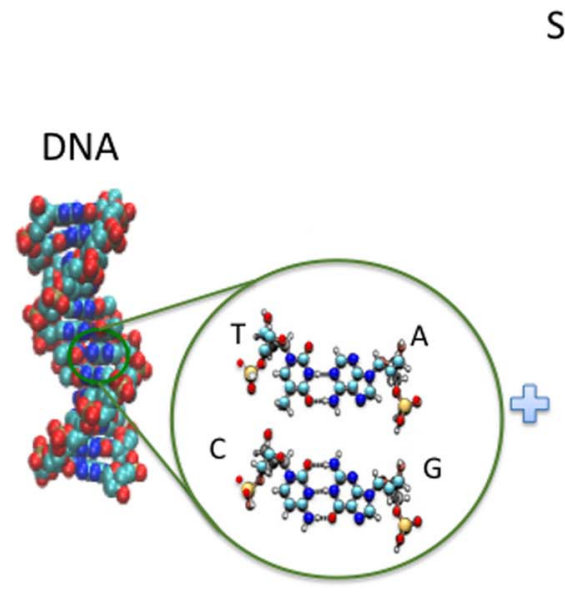

(a)

\section{SWCNT}

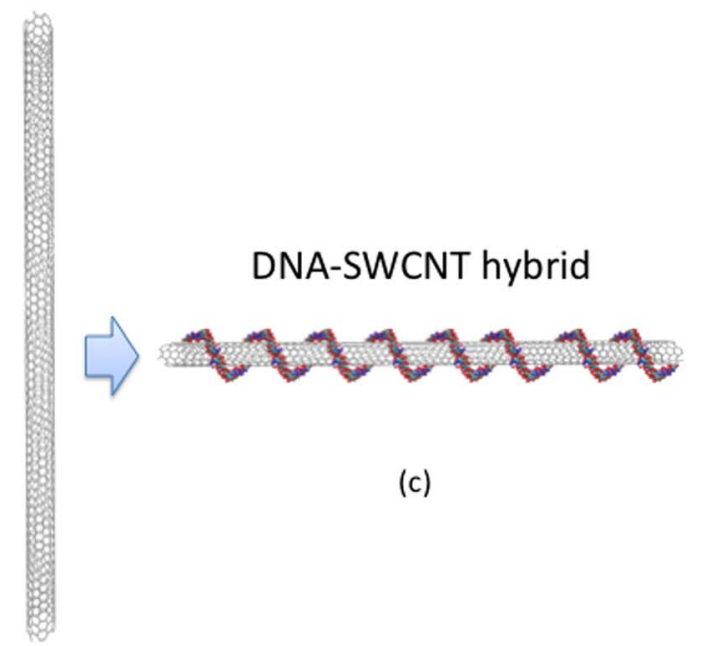

(b)

Figure 1. DNA-SWCNT hybrid structure. (a) The structural model of deoxyribonucleic acid (DNA), (b) a single-walled carbon nanotube (SWCNT), and (c) the DNA-SWCNT complex.

\section{SWCNT Molecular and Fluorescence Properties for Sensing Applications}

Since the discovery of SWCNT fluorescence, multiple research groups have focused on exploiting their exceptional optical properties for the construction of a new generation of sensing devices. The SWCNT near-infrared fluorescence emission is directly related to SWCNT structure. SWCNTs are carbon-based, tubular 1-D nanostructures that belong to the family of synthetic carbon allotropes (e.g. diamond, graphite, fullerenes, etc.) obtained by rolling a graphene sheet into a cylindrical shape. Depending on the rolling direction, which is defined by the chiral index $(n, m)$, a range of SWCNT chiralities differing in length, diameter, roll-up angle, and bandgap are possible. ${ }^{21}$ These chiralities can be schematically categorized as either metallic (bandgap $0 \mathrm{eV}$ ), semimetallic (bandgap $\approx 1-100 \mathrm{meV}$ ), or semiconducting (bandgap $\approx 0.5-1 \mathrm{eV}$ ). ${ }^{22}$ Metallic and symimetallic SWCNTs possess achiral symmetries (armchair $(n, n)$ and zigzag $(n, 0)$ nanotubes) that do not fluoresce. On the other hand, semiconducting SWCNTs are characterized by chiral structures with intrinsic nIR fluorescence emissions. Semiconducting SWCNTs absorb light at $\mathrm{E}_{22}$ excitation energies and emit fluorescence at $\mathrm{E}_{11}$ transitions in the nIR wavelength range between 900 and $1600 \mathrm{~nm}$. When a SWCNT is photo-excited, electron-hole pairs with a certain coulombic interaction are generated to form excitons. ${ }^{23}$ As excitons move along the carbon nanotube surface, they are influenced by perturbations in the chemical environment, such as molecules binding to the nanotube surface. ${ }^{24}$ The possibility of modulating semiconducting nanotube emission in response to specific molecular interactions is the focus of current endeavors in developing novel sensing elements for disparate applications.

To be used in biological fluorescence sensing platforms, SWCNTs need to be suspended in aqueous or organic solvents. Unfortunately, due to their inherently hydrophobic surfaces, pristine carbon nanotubes are practically insoluble. ${ }^{25}$ Furthermore, bundled SWCNTs do not significantly emit nIR fluorescence because of energy transfer to neighboring tubes. ${ }^{26}$ Therefore, surfactant- (e.g. sodium dodecyl sulfate (SDS), sodium dodecylbenzenesulfonate (SDBS), or sodium cholate) or polymer- (e.g. biopolymers or synthetic polymers) assisted sonication of SWCNT bundles ${ }^{27}$ is often used to improve SWCNT dispersions in aqueous or organic solvents. This approach benefits from the non-covalent functionalization of the nanotube surface, which largely preserves the nanotube's optical properties compared to covalent surface modifications that may result in fluorescence loss. ${ }^{28,29}$

Among the different dispersion materials, DNA possesses beneficial structural characteristics for coating and separating bundled nanotubes in aqueous solutions often with higher efficiencies compared to other types of surfactants. ${ }^{30}$ The resulting DNA-SWCNT complexes that form from DNA interaction with the SWCNT surface are the result of consorted interactions between the hydrophobic and electron-rich nanotube surface, the aqueous surrounding, and the chemically diverse DNA oligonucleotides. In these dynamic structures, four types of aromatic nitrogenous bases, two purines (adenine (A) and guanine $(\mathrm{G})$ ) and two pyrimidines (cytosine (C) and thymine $(\mathrm{T})$ ), interact with the nanotube sidewalls, allowing the DNA sugar-phosphate backbone to be exposed to the aqueous surrounding ${ }^{17,31-34}$ (Figure 1). Because of the hydrophilic nature of the DNA phosphate backbone, the DNA-SWCNT complex is highly soluble in aqueous media. ${ }^{35}$

The mechanism of DNA wrapping on SWCNTs depends on multiple physical contributions. In particular, entropy loss due to the confinement of the DNA backbone, van der Waals and hydrophobic interactions between DNA nucleobases, as well as the electrostatic interactions between charged residues play a notable role in the dispersion process. ${ }^{36-37}$ Despite their non-covalent nature, all these forces contribute to strengthen DNA immobilization onto the nanotube's sidewalls, and the resulting solutions can be stable for months at room temperature. ${ }^{20,38}$ In addition to facilitating mono-dispersion and enhancing biocompatibility, DNA interaction with the SWCNT surface represents one of the most effective ways to provide carbon nanostructures with analyte selectivity.

The first demonstration of non-covalent DNA immobilization on carbon nanotubes was done by Tsang and coworkers in $1997 .{ }^{39}$ In the following years, this topic has drawn incredible attention as evidenced by the large number of publications focused on understanding DNA-carbon nanotube interactions and establishing a more detailed definition of possible binding geometries and orientations in the hybrid systems. ${ }^{40}$ Effective immobilization of the molecular recognition elements is essential for ensuring the highest possible efficacy of the sensing surface. Furthermore, as principal mechanisms behind the DNA nanoscale interactions become clearer, a better understanding of the molecular recognition mechanisms may be possible to an extent that would allow researchers to even predict sensor selectivities. The following paragraphs will discuss the factors contributing to DNASWCNT conjugation mechanisms in greater detail.

\section{Molecular-level Interactions of DNA-SWCNT Hybrids}

Both theoretical simulations and experimental investigations have greatly contributed to the development of several models for 
describing DNA-nanotube interactions at the nanoscale. ${ }^{37,41-46}$ These models have been used to study SWCNTs interacting with oligomers of varying lengths (from $\sim 0$ bases $^{30,47}$ to $\sim 100$ bases) for both singlestranded DNA (ssDNA) ${ }^{48-49}$ and double-stranded DNA (dsDNA), ${ }^{48}$ as well as hybrids consisting of both alternating ssDNA and dsDNA. ${ }^{41}$ Changing the length and sequence of the DNA, as well as the SWCNT diameter and chirality, can strongly affect the dispersion process. ${ }^{30}$ Among homo-oligonucleotides based on 60-nucleobases, researchers demonstrated that thymine-based sequences have the highest dispersion yields. Simulations have confirmed that $\pi-\pi$ stacking interactions between nucleic acid bases of poly(T)-ssDNA and the nanotube surface represent the basic forces governing DNA-SWCNT conjugation, favorably contributing to the stability of the resulting hybrids. As expected, the hydrophilic sugar-phosphate backbone orientation toward the aqueous surrounding accounts for the high solubility in water. Furthermore, when testing the effects of different lengths of $\mathrm{d}(\mathrm{T})_{\mathrm{n}}$-DNA (where $\mathrm{n}=15,21,30$ and 60 ) on solubilization and separation, the $\mathrm{T}_{30}$ motif was shown to possess the highest dispersion efficiency. ${ }^{30}$ A subsequent report in 2007 demonstrated that short DNA strands possess excellent dispersion yields in comparison to longer oligodeoxynucleotides, and a mixture of the complementary oligonucleotides $\mathrm{d}(\mathrm{GT})_{3}-\mathrm{d}(\mathrm{AC})_{3}$ may result in the highest solubilization efficiency among the sequences studied. Kinetic measurements suggest that the ssDNA-SWCNT complexes are more stable than the DNA-DNA duplexes formed between short- and medium-sized DNA molecules ${ }^{50}$ Hughes and co-workers (2010) also studied the effect of short DNA oligomers such as $\mathrm{A}_{15}, \mathrm{G}_{15}, \mathrm{C}_{15}$, and $\mathrm{T}_{15}$ on the dispersion of SWCNTs. Photoluminescence and absorption measurements were used to define a precise order of dispersion efficiency among the different nucleobases, $\mathrm{T}>\mathrm{C}>\mathrm{G} \gg \mathrm{A}$, with the thymine-based DNA strands once more demonstrating the best debundling efficiencies and cytosine-based sequences having the fastest adsorption kinetics. ${ }^{51}$

A comparison of the adsorption affinities of ssDNA and dsDNA suggests that the former is capable of creating more stable DNASWCNT complexes with the latter demonstrating a much weaker affinity for the carbon sidewalls. Such differences in the adsorption patterns arise from structural and chemical differences between ssDNA and dsDNA. ${ }^{34}$ In dsDNA, the alternating nucleobases exist in a highly hydrophobic environment that is confined inside the double-helical structure while the sugar-phosphate backbone allows the dsDNA molecule to interact freely with the hydrophilic environment. ${ }^{34-35}$ On the other hand, ssDNA possesses hydrophobic bases that are freely available to interact with the hydrophobic surface of the SWCNT. ${ }^{30,34}$ Molecular dynamic simulations have shown that the ssDNA has many degrees of freedom and a rugged free-energy landscape containing many local minima. ${ }^{42}$ These characteristics result in a more flexible structure than dsDNA, whose double helix structure is more rigid..$^{30,34-35}$ The differences are more clearly seen when comparing the binding characteristics of a solution containing a combination of both single- and double-stranded DNA. Gladchenko and co-workers used an AMBER Force Field to study the binding interactions between ssDNA-dsDNA hybrids and $(16,0)$ SWCNTs. The ssDNA-dsDNA hybrid consisted of a 35-nucleotide polyd $(A) d(T) d(G) d(C)$ sequence with a complementary 5-nucleotide sequence connected to the central part of the motif ${ }^{41}$ (Figure 2). The results suggest that the single-stranded portions create a sort of "anchor" on the SWCNT sidewalls for attaching the whole polymer.

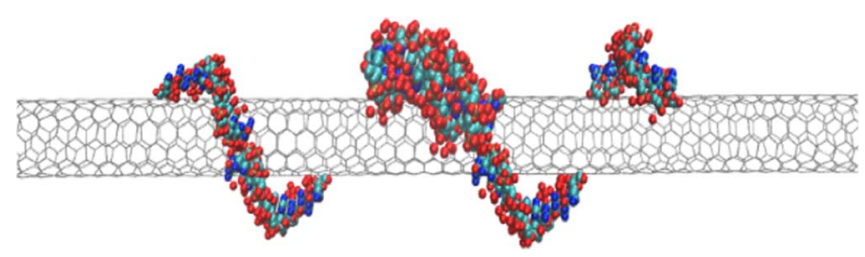

Figure 2. (16,0) SWCNT wrapped by a ssDNA-dsDNA hybrid with a 35nucleotide sequence (ATGC) and 5-nucleotide complementary fragment.

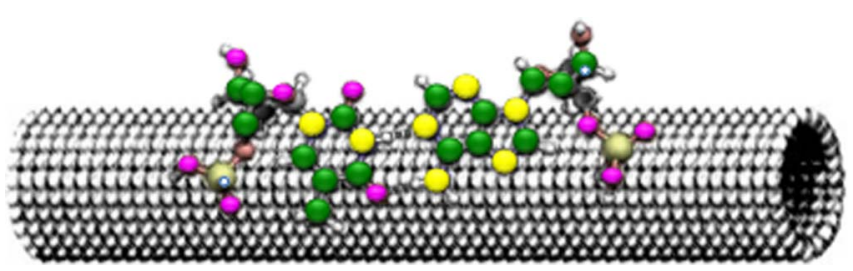

Figure 3. Schematic representation of the nucleic acid base-SWCNT interaction.

These portions are highly flexible, increasing the freedom of rotation for the sugar-phosphate backbone. The double-stranded region interacts with SWCNT sidewalls through electrostatic forces, keeping the DNA closer to the SWCNT surface. Interestingly, approximately $60 \%$ of the bases were found not to stack with the nanotube, with no stacking interaction in the double-stranded region. This might occur because of the steric hindrance between alternating different-sized purine and pyrimidine nucleotides which, despite the high flexibility of the sugar backbone, could not easily position along the nanotube surface.

In addition to elucidating the interaction forces responsible for DNA immobilization on SWCNTs, theoretical approaches have been helpful in predicting the characteristic binding geometry of DNASWCNT systems. For example, CHARMM calculations carried out by Meng et al. were used to analyze the localized interaction of a single nucleotide adsorbed on the SWCNT surface to determine energetically favorable configurations of the bases on the nanotube sidewall. ${ }^{40}$ The orientation of a base with respect to the tube axis can be very different, especially when interacting with highly asymmetric nanotube structures. The nucleotide tends to bind the carbon nanotube through its base unit located $3.3 \AA$ away from the SWCNT sidewall. The most stable base orientation is formed when the sugar-base points perpendicularly or slightly tilted to the tube axis. Whereas the base unit remains planar without significant bending, the sugar residue is more flexible. ${ }^{35}$ The parallel configuration of the bases is obtained because it maximizes the van der Waals interaction between the carbon nanotube sidewall and the molecule. ${ }^{52}$

The interaction energy between the aromatic nucleic bases and carbon-based nanotube surface is related to the size of the purine and pyrimidine bases. In comparison to pyrimidines, purines have an additional five-member imidazole ring in their structures that increases the interaction surface are $^{53}$ (Figure 3 ). Consequently, the $\pi$-stacking interactions between adenine/guanine with SWCNTs are stronger than the cytosine/thymine interactions. As described by Hunter et al., ${ }^{54}$ the $\pi-\pi$ stacking energy should increase with increasing surface area of the faces of the interacting partners, which was also confirmed by several research groups. ${ }^{43,52,55-56}$ For example, Johnson et al. ${ }^{43}$ showed that the binding energies of adenine and guanine, which contain two aromatic rings, with the $(11,0)$ SWCNT are higher than those of the single-aromatic ring bases, cytosine and thymine. The binding energies of adenine, guanine, cytosine, and thymine were found to be $0.60,0.65,0.48$ and $0.55 \mathrm{eV}$, respectively. This result has been confirmed in an alternative study ${ }^{57}$ that used DFT calculations to determine the interaction energies of nucleic acid bases with the small diameter $(\sim 5.5 \AA)(7,0)$ SWCNT. Once again, guanine was predicted to have the highest binding energy.

A hybrid DFT approach was also used by Wang ${ }^{55}$ to investigate the self- and cross-stacking of four DNA bases with the $(5,5)$ SWCNT and $(10,0)$ SWCNT in both the gas phase and aqueous solution. In the gas phase, guanine was shown to have the highest binding energy for self- and cross-stacking. The self-stacking of the nucleobases was found to decrease in the order $\mathrm{G}>\mathrm{A}>\mathrm{C}>\mathrm{T}$, while cross-stacking varied in the order $\mathrm{G}>\mathrm{A}>\mathrm{T}>\mathrm{C}$ for both $(10,0)$ and $(5,5)$ SWCNTs. Conversely, for aqueous solutions, the binding preferences varied from $\mathrm{A}>\mathrm{G}>\mathrm{T}>\mathrm{C}$ for $(10,0)$ SWCNTs to $\mathrm{G}>\mathrm{A}>\mathrm{T}>\mathrm{C}$ for $(5,5)$ SWCNTs. The different binding energies in aqueous solutions compared to the gas phase are due to the strong solvation tendency of 


\begin{tabular}{|c|c|c|c|}
\hline Base & $E_{\mathrm{b}}{ }^{\mathrm{CNT}}(\mathrm{eV})$ & $E_{\mathrm{b}}{ }^{\text {graphene }}(\mathrm{eV})$ & $\alpha\left(e^{2} a_{0}^{2} E_{\mathrm{h}}^{-1}\right)$ \\
\hline G & 0.49 & 0.61 & 131.2 \\
\hline A & 0.39 & 0.49 & 123.7 \\
\hline $\mathrm{T}$ & 0.34 & 0.49 & 111.4 \\
\hline $\mathrm{C}$ & 0.29 & 0.49 & 108.5 \\
\hline $\mathrm{U}$ & 0.28 & 0.44 & 97.6 \\
\hline
\end{tabular}

nucleobases, which significantly affects the interaction between the aromatic nucleobases and the SWCNTs. Das and co-workers applied the ab initio Hartree-Fock approach to analyze base binding energies with the $(5,5)$ SWCNT, both accounting and not accounting for the solvation energy. ${ }^{52}$ The rank order of the binding energies without accounting for water solvation was found to be $\mathrm{G}>\mathrm{A}>\mathrm{T}>\mathrm{C}$. When accounting for the solvation effect, the relative binding energy preferences changed from $\mathrm{G}>\mathrm{A}>\mathrm{T}>\mathrm{C}$ to $\mathrm{G}>\mathrm{T}>\mathrm{A}>\mathrm{C}$, as confirmed by experimental studies.

Interestingly, this study also demonstrated that the distinct curvature of carbon nanotubes can also have an important effect on the binding energies of the nucleobases. In particular, increased curvature was shown to result in a lower binding efficiency, as confirmed by Gowtham et al. ${ }^{53}$ As presented in the Table I, purine bases, guanine $(0.49 \mathrm{eV})$ and adenine $(0.39 \mathrm{eV})$, possess the highest binding energies. The molecular polarizability $(\alpha)$ of the base molecules has also been shown to play a dominant role in the interaction strength of the nucleic acid base with the SWCNT.

Experimental measurements have been used to study the overall structure of DNA-SWCNT hybrids that result from these individual nucleotide binding contributions. For example, atomic force microscopy (AFM) images clearly show ssDNA helically wrapping a single SWCNT with a constant periodicity along its axis ${ }^{58}$ (Figure 4). Transmission electron microscopy (TEM) images of DNA-SWCNTs further verify the helicity of the DNA relative to the individual nanotube axis. However, despite the valuable mechanical and topological information AFM and TEM measurements provide about the DNA wrapping, these measurements were performed in the solid phase, in contrast to the aqueous dispersions predominantly used during optical measurements. This disparity in the nanotube environment limits the interpretation of these measurements with regard to the DNA-SWCNT structure in solution. For example, in addition to adsorption and charge-based conformational confinement, DNA-SWCNTs prepared on solid-state substrates such as mica for AFM imaging undergo heating and cooling procedures during deposition that may perturb the DNA-SWCNT interaction. ${ }^{59}$ In addition to limitations on data interpretation, these measurements have limited sensitivities. For example, the nano-Newton AFM force resolution is too high to accurately measure the pico-Newton DNA-SWCNT interactions, particularly in the presence of exogenous contributions from charged substrates such as mica.

Despite these limitations, the overall wrapping helicity observed with AFM has been predicted by theoretical models suggesting that electrostatic and torsional interactions within the sugar-phosphate backbone serve as the primary driving force behind $3^{\prime}$ to $5^{\prime}$ ssDNA self-assembly. ${ }^{43,60-61}$ These studies suggest that many stable ssDNASWCNTs structures exist, ${ }^{35}$ including the stable horseshoe structure that is observed for other oligomers such as poly $(\mathrm{dG})_{6}$ and poly $(\mathrm{dC})_{6}$. Other stable structures include the DNA strand linearly aligning along the nanotube axis, forming an S-shaped structure on the SWCNT sidewall, or contributing to a helix structure. The results of molecular dynamics simulations by Johnson et al. ${ }^{62}$ suggest a variety of possible DNA binding geometries, including right- and left-handed helical wrappings as well as more disordered structures. Martin and co-workers also discovered a variety of distinct geometries that de-
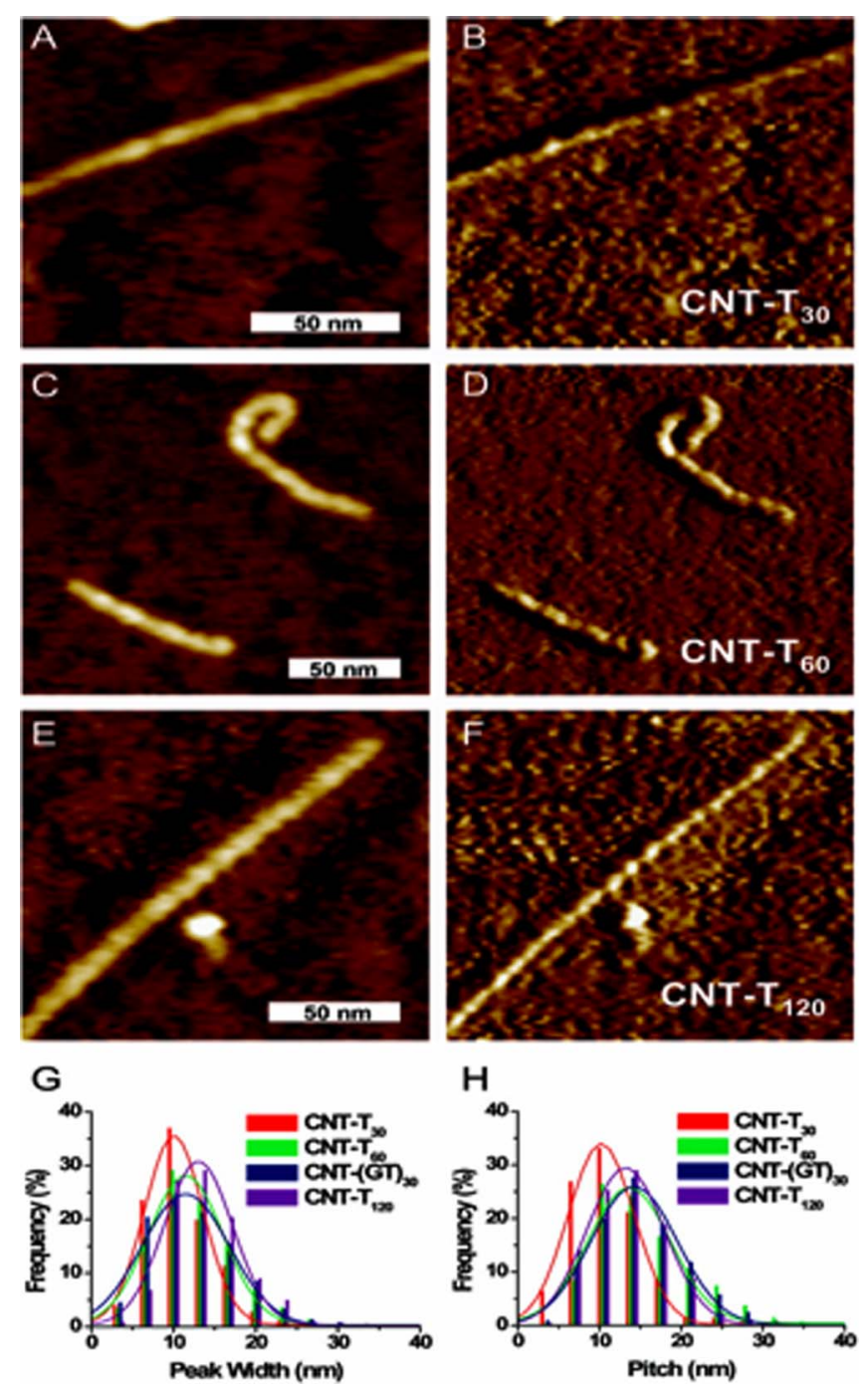

Figure 4. (A-F) AFM height (left) and phase (right) images of representative SWCNTs wrapped with thymine oligonucleotide sequences of 30-, 60-, and 120-base length (shown with 5-nm height scales and phase scales of $8^{\circ}, 25^{\circ}$, and $10^{\circ}$, respectively). (G) Peak width along the SWCNT surface and $(\mathrm{H})$ pitch (peak-to-peak distance) distributions for SWCNTs wrapped with $\mathrm{T}_{30}, \mathrm{~T}_{60}$, $(\mathrm{GT})_{30}$, and $\mathrm{T}_{120}$ oligonucleotides $(n>500)$. Reproduced with permission from Ref. 58 .

pend on the DNA sequence and SWCNT diameter and chirality. ${ }^{44}$ It is important to note that like the experimental AFM and TEM measurements, these computational predictions have limitations. For example, molecular dynamics simulations offer nano-second timescales that are too short to simulate experimental observations that may occur on the second timescale.

The ability of DNA to form chirality-specific, well-ordered structures around distinct $(n, m)$ nanotube species has enabled its use for chirality separation applications through ion-exchange chromatography and more recently through polymeric aqueous two-phase (ATP) extraction. ${ }^{63}$ Researchers have identified more than 20 40-mer DNA sequences with periodic purine-pyrimidine patterns that are capable of selectively recognizing distinct SWCNT chiralities by forming a specific three-dimensional barrel motif through inter- and intra-strand hydrogen bonding. ${ }^{64}$ By applying replica exchange molecular dynamics (REMD) to study the equilibrium structures formed by ss-DNA of varying sequences on a $(6,5)$ SWCNT, Roxbury et al. have shown the molecular basis of the sequence dependency of SWCNT sorting to be strongly influenced by the variable nature of base-base hydrogen bonding. ${ }^{65}$ For example, the DNA sequence (TAT) ${ }_{4}$ is shown to form a right-handed helically wrapped barrel that is stabilized by 

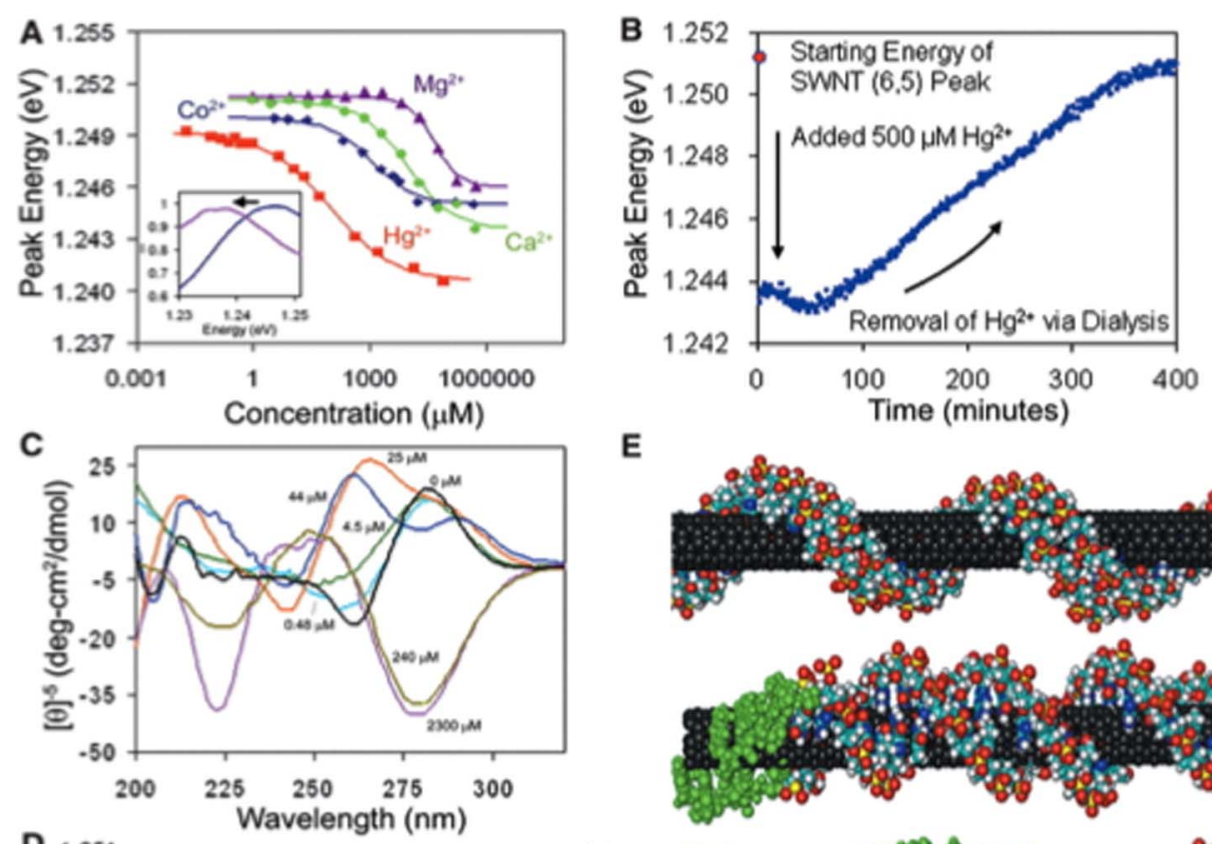

$\mathbf{E}$
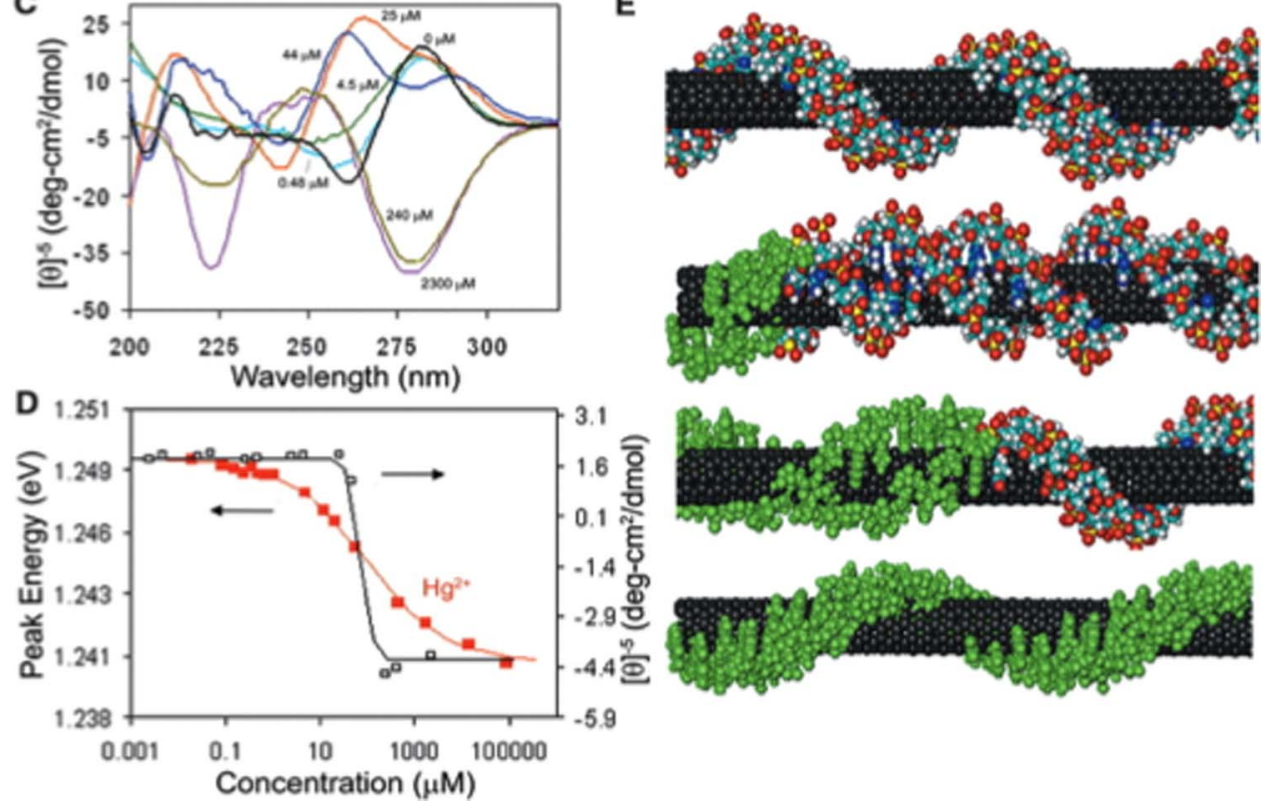

Figure 5. (A) Concentration-dependent fluorescence response of the DNA-encapsulated (6,5) nanotube to divalent chloride counterions. The inset shows the (6,5) fluorescence band at starting (blue) and final (purple) concentrations of $\mathrm{Hg}^{2+}$. (B) Fluorescence energy of DNA-SWCNTs inside a dialysis membrane upon removal of $\mathrm{Hg}^{2+}$ during a period of 7 hours by dialysis. (C) Circular dichroism spectra of unbound $\mathrm{d}(\mathrm{GT})_{15}$ DNA at various concentrations of $\mathrm{Hg}^{2+}$. (D) DNA-SWCNT emission energy plotted versus $\mathrm{Hg}^{2+}$ concentration (red curve) and the ellipticity of the 285 -nm peak obtained via circular dichroism measurements upon addition of mercuric chloride to the same oligonucleotide (black curve). Arrows point to the axis used for the corresponding curve. (E) Illustration of DNA undergoing a conformational transition from the $\mathrm{B}$ form (top) to the $\mathrm{Z}$ form (bottom) on a carbon nanotube. Reproduced with permission from Ref. 48.

intra-strand, self-stitching hydrogen bonding. The same sequence forms a different, less-stable structure on the larger diameter $(8,7)$ SWCNT, providing evidence for SWCNT selectivity. ${ }^{66}$ Other factors such as temperature and ultrasonication can also affect DNA-SWCNT hybrid morphology. For example, increasing the temperature allows the base to more easily deviate from the optimal adsorption position, promoting detachment from the SWCNT wall. ${ }^{35}$

\section{Engineering Sensor Selectivity of NIR Fluorescence Sensors Based on DNA-SWCNTs}

We have explained how theoretical and experimental investigations can help in elucidating the underlying mechanisms and overall geometries formed from DNA immobilization on the hydrophobic nanotube sidewalls. However, the role of DNA conformation and binding geometry in engineering a selective fluorescence response toward specific analytes of interest remains unclear. In this section, we highlight the contributions that have been identified thusfar in explaining the observed DNA-SWNT sensor selectivity.

Sequence-complementarity is the primary factor considered when studying ssDNA-SWCNT specificity toward target oligonucleotide strands. As a direct consequence of the DNA's intrinsic ability for complementary base-pairing, ssDNA wrappings have found immediate application for the study of DNA hybridization as well as the detection of single-nucleotide genomic mutation events. Jeng et al. ${ }^{38}$ have shown that coupling ssDNA motifs around SWCNTs with their corresponding complements results in the optical modulation of the near-infrared fluorescence, specifically resulting in a solvatochromic shift following DNA hybridization. The discovery of this phenomenon has been applied to detect conformational and single-nucleotide polymorphisms (SNPs). For example, Jeng et al. designed a SNP sensor by preparing a suspension of $(6,5)$ SWCNTs embedded with a specific ssDNA sequence (5'-TAG CTA TGG AAT TCC TCG TAG GCA -3'). Upon exposure to either a complementary DNA strand or a sequence containing a SNP, they observed significant differences in the SWCNT emission energies that were consistent with only partial hybridization of the SNP strand lacking full DNA complementarity. ${ }^{67}$

DNA conformational changes upon interaction with divalent metal cations (e.g. $\mathrm{Hg}^{2+}, \mathrm{Co}^{2+}, \mathrm{Ca}^{2+}$, and $\mathrm{Mg}^{2+}$ ) were investigated by Heller and co-workers in 2006 using ssDNA oligonucleotides with a repeating G-T sequence ( $\mathrm{GT}_{15}$-DNA) to wrap SWCNTs (Figure 5). Exposure of the DNA-SWCNTs to complementary DNA strands in the presence of increasing ionic concentrations allowed the authors to detect double-stranded DNA gradual switches from B-form to a more irregular Z-form configuration. Using this platform, they detected the B-Z transition in whole blood, tissue, and live mammalian cells. ${ }^{48}$

Although sequence-dependency plays a clear role in the detection of complementary strands, its contribution toward detecting other molecular species and biological analytes, such as NO, is not as predictive. By screening an array of nIR fluorescent semiconducting 

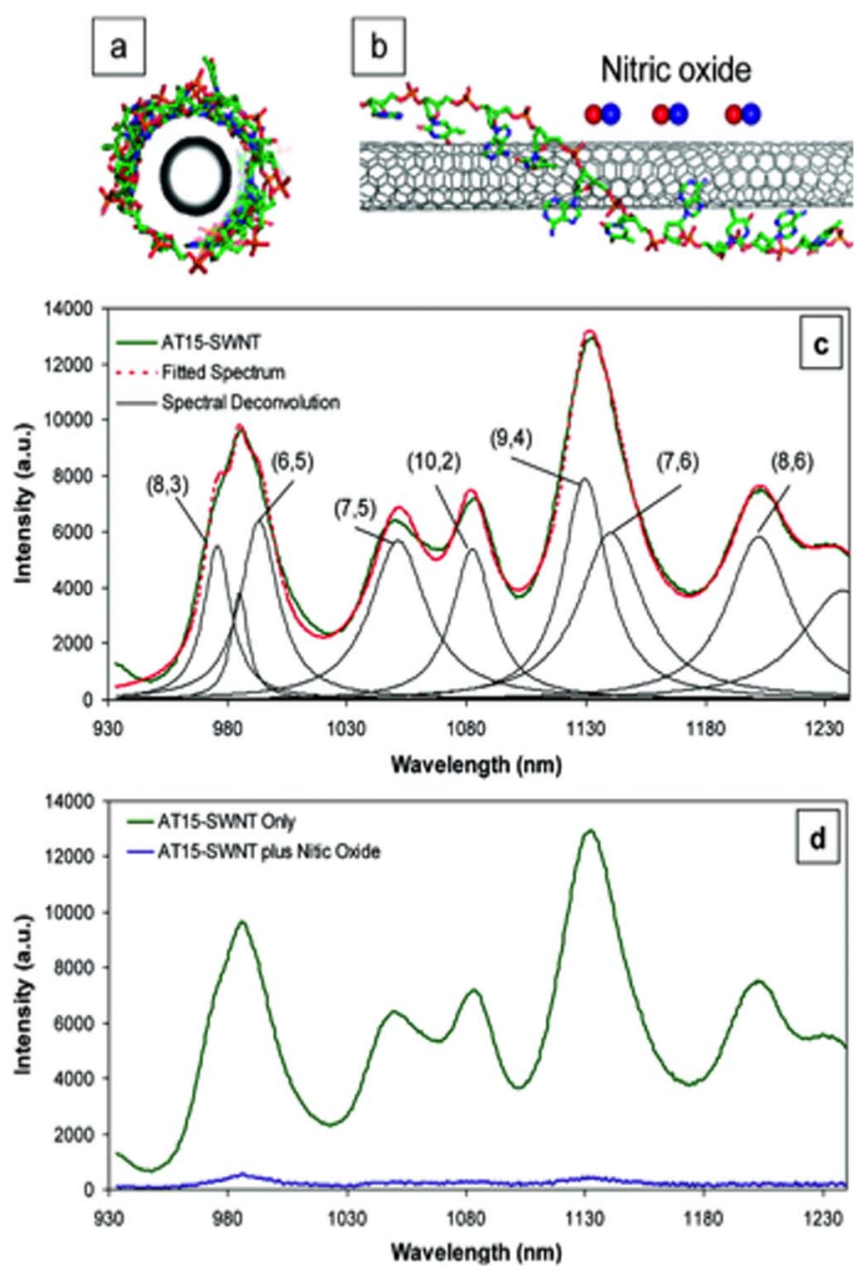

Figure 6. The optical response of $\mathrm{AT}_{15}$-SWCNTs upon exposure to NO. (a) Front and (b) side schematic views of one of the several binding structures of $\mathrm{AT}_{15}$-SWCNT simulated using HyperChem. DNA bases stack on the sidewall of the SWCNT, and the sugar-phosphate backbone extends away from the surface. (c) The nIR fluorescence spectrum (solid green) of $\mathrm{AT}_{15}-\mathrm{SWCNT}$ recorded with a $50 \times$ objective upon $785 \mathrm{~nm}$ laser excitation $(150 \mathrm{~mW}$ at the sample). The spectral deconvolution reveals seven nanotube species and a Raman peak (solid black), with the sum of these contributions (dotted red) matching the profile of the actual data. (d) Complete quenching (solid blue) of $\mathrm{AT}_{15}-\mathrm{SWNT}$ fluorescence is observed when the SWCNT sample is exposed to NO $(60 \mu \mathrm{M}$, in 1x PBS). Reproduced with permission from Ref. 68.

SWCNTs wrapped in 30-base oligomers with varying sequences, Zhang and co-workers exemplified the ability of DNA-based nanoplatforms to selectively recognize specific redox target molecules, such as NADH, melatonin, and L-ascorbic acid. ${ }^{68}$ Among several factors hypothesized to play a relevant role, redox selectivity is one of the most likely to be addressed. The authors explained this peculiar capability by considering the relative positions of the LUMO levels of the DNA wrappings with respect to the HOMO levels of the tested reducing agents and the Fermi levels of semiconducting SWCNTs. Competition between the HOMO electrons of each tested compound and the excited-state electrons of the SWCNT for the vacancies in the LUMO level of DNA was believed to account for the observed fluorescence enhancement. As further confirmation of this mechanism, decreasing of the energy gap between the conduction band of SWCNTs and the LUMO level of DNA resulted in a decrease in the fluorescence changes. Altogether, these observations offer a promising approach to engineering novel recognition elements by simply modulating the redox potentials of the wrappings.

However, although redox properties may account for certain molecular specificities, they alone cannot not account for all sensor selectivities. For example, contrary to the unfavorable predicted redox interactions, Zhang et al. observed that SWCNT complexes functionalized with a DNA motif containing 30 alternating A-T bases exhibited a strong optical response toward single NO molecules (Figures 6). ${ }^{68}$ One possible explanation for this selectivity involved contributions from steric interactions. The DNA may form a specific conformation when wrapping around the nanotube sidewalls in a manner that results in the exclusion of most species from direct contact with the nanotube, allowing only small target analytes to access the nanotube surface. Compared to alternative DNA sequences, the formation of more closely spaced AT bands along the circumference of the SWCNT could selectively block the adsorption of molecules greater in size than NO..$^{59,68}$ This hypothesis is supported by the fact that (AT) $)_{15}$-SWCNT fluorescence was unaffected in the presence of larger molecules such as dopamine, melatonin, NADH, and L-ascorbic acid, allowing these sensors to be used in complex biological environments such as A375 melanoma cells for measuring the intracellular spatial and temporal distribution of NO. ${ }^{69}$ Beyond bioanalyte detection, DNA-SWCNTs have also been used to detect synthetic chemical moeities including nitroaromatics. For example, Heller et al. have demonstrated that electronic and steric effects of the same encapsulating $(\mathrm{AT})_{15}$ DNA sequence can impart SWCNTs with specific selectivities toward a wide range of nitroaromatics, including trinitrotoluene (TNT) ${ }^{70}$ Alternative DNA sequences have also been used to detect a range of analytes. In 2014 , Kruss et al. showed that $(\mathrm{GT})_{15}$ DNA and $(\mathrm{GU})_{15}$ RNA-wrapped SWCNTs can serve as highly selective and sensitive fluorescence turnon sensors for catecholamine neurotransmitters such as dopamine. ${ }^{71}$ Their results suggest that DNA-SWCNTs can act as conformational switches that reversibly modulate SWCNT fluorescence. ${ }^{59}$

Landry and co-workers have extended this platform to study ribonucleic acid (RNA) contributions to fluorescence response. ${ }^{72}$ Recent experimental measurements suggest that the differences in $(\mathrm{GU})_{15}$-RNA and (GT) $)_{15}$-DNA conformations have a significant impact on fluorescence response. A comparison of both polymers shows that the $(\mathrm{GU})_{15}$-RNA has a lower conformational stability on the SWCNT than the corresponding DNA-based complex. In the case of DNA, most nucleotides stack onto the SWCNT surface, whereas only a few bases of the RNA strand bind to the SWCNT. Different SWCNT fluorescence responses were observed as a consequence of this disparity.

Specific analyte reactions to particular DNA binding sites may also account for the sequence-dependency of the fluorescence modulation of SWCNT sensors. For instance, the selective interaction of DNA-wrapped nanotubes with reactive oxygen species (ROS) and alkylating agents was reported in 2009 by Heller and co-workers, ${ }^{73}$ who provided one of the first examples of multimodal optical SWCNT sensors. The reaction mechanism of this multimodal sensor is based on four reaction pathways between the different analytes and the d(GT) $)_{15}$ DNA-bound SWCNTs. The selective reaction of the chemotherapeutic alkylating agent melphalan with guanine nucleobases has been shown to result in a uniform redshift in the PL bands of the $(6,5)$ and $(7,5)$ nanotubes. Analogously, singlet oxygen-induced DNA adducts resulting from nucleobase oxidation due to $\mathrm{Cu}^{2+}$ and $\mathrm{H}_{2} \mathrm{O}_{2}$ exposure caused a pronounced redshift of the $(6,5)$ nanotube emission. ${ }^{73}$ In an alternative reaction, the direct adsorption of $\mathrm{H}_{2} \mathrm{O}_{2}$ onto the nanotube resulted in the attenuation of the nanotube emission and a slight concomitant energy shift. DNA damage induced by hydroxyl radicals produced in the presence of $\mathrm{Fe}^{2+}$ and $\mathrm{H}_{2} \mathrm{O}_{2}$ also attenuates emission, preferentially affecting the $(7,5)$ chirality in the absence of energy shifts.

Sen et al. ${ }^{74}$ also used a d(AT) $)_{15}$-SWCNT complex to detect multiple reactions of riboflavin with ROS and Trolox. In this study, the SWCNT emissions were quenched by riboflavin-induced ROS formation. The complex reaction network and opposing contributions of the different reaction products to the SWCNT fluorescence reaction mechanism resulted in an oscillatory fluorescence response. In an alternative platform, Cha et al. used a 30-base DNA sequence containing tandem motifs of G-rich units that are capable of forming unique secondary structures called G-quadruplexes to coat the 
nanotube's surface and enable exquisite selectivity toward insulin molecules. The peculiar aptamer quadruplex conformation was preserved after SWCNT functionalization, suggesting selective photoluminescence quenching through a photoinduced charge transfer mechanism. ${ }^{75}$

\section{Conclusions}

Since the discovery of the intrinsic SWCNT nIR fluorescence, researchers in the community have focused on exploiting the beneficial photoluminescent properties to develop novel biosensing platforms. Current endeavors have been devoted to engineering specific wrappings capable of enriching the nanotube surface with exquisite recognition capabilities toward a wide range of different analytes. DNA-wrapped SWCNTs have emerged as the most studied SWCNTbased probe, benefiting from the versatility of engineered DNA coupled with the high sensitivity, robustness, and biocompatibility of engineered SWCNTs to yield composite sensing architectures with improved performance. The primary forces that govern the DNASWCNT interaction has been studied using both theoretical and empirical measurements, and these techniques have been used to significantly advance the underlying understanding of the binding affinities, wrapping geometry, and single nucleobase orientation onto nanotube sidewalls. This understanding has been used to identify the primary factors influencing the resulting molecular recognition behavior. For instance, sequence complementarity, as well as redox properties and steric interactions, have been found to play an important role in modulating DNA-SWCNT fluorescence in response to specific analytes of interest, enabling their highly selective detection even in the presence of competing analytes in complex biological environments. Combined with the insightful contributions offered from modern computational methods, such empirical observations provide a powerful approach toward advancing the fundamental understanding behind these peculiar molecular moeities. The ongoing investigations in the field serve to not only further this understanding, but also hold promise toward achieving the biosensing gold standard of rationally designing sensing probes with pre-defined selectivities.

\section{References}

1. L. C. Clark Jr. and C. Lyons, Ann. N. Y. Acad. Sci., 102, 29 (1962).

2. F.-G. Banica, p.1, John Wiley \& Sons, Chichester (2012).

3. M. R. Leyden, R. J. Messinger, C. Schuman, T. Sharf, V. T. Remcho, T. M. Squires, and E. D. Minot, Lab on a Chip, 12, 954 (2012).

4. R. Kellner, J.-M. Mermet, M. Otto, M. Valcárcel, and H. M. Widmer, p. 1, WILEY$\mathrm{VCH}$, Weinheim (2004).

5. K. Yum, T. P. McNicholas, B. Mu, and M. S. Strano, J. Diabetes Sci. Technol. 7, 72 (2013).

6. R. Saito, G. Dresselhaus, and M. S. Dresselhaus, Physical Properties of Carbon Nanotubes, p. 35, ICP Imperial College Press, London (1998).

7. M. J. O'Connell, S. M. Bachilo, C. B. Huffman, V. C. Moore, M. S. Strano, E. H. Haroz, K. L. Rialon, P. J. Boul, W. H. Noon, C. Kittrell, J. Ma, R. H. Hauge, R. B. Weisman, and R. E. Smalley, Science, 297, 593 (2002).

8. D. A. Heller, S. Baik, T. E. Eurell, and M. S. Strano, Adv. Mater. 17, 2793 (2005).

9. S. M. Bachilo, M. S. Strano, C. Kittrell, R. H. Hauge, R. E. Smalley, and R. B. Weisman, Science, 298, 2361 (2002).

10. R. Weisslder, Nat. Biotechnol. 19, 316 (2001).

11. R. Elghanian, Science, 277, 1078 (1997).

12. J. M. Nam, C. S. Thaxton, and C. A. Mirkin, Science, 301, 1884 (2003)

13. M. J. O'Connell, P. Boul, L. M. Ericson, C. Huffman, Y. Wang, E. Haroz, C. Kuper, J. Tour, K. D. Ausman, and R. E. Smalley, Chem. Phys. Lett. 342, 265 (2001).

14. J. D. Watson and F. H. C. Crick, Nature, 171, 737 (1953).

15. M. H. F. Wilkins, A. R. Stokes, and H. R. Wilson, Nature, 171, 738 (1953).

16. R. Franklin and R. G. Gosling, Nature, 171, 740 (1953).

17. S.-H. Lee and C. Mao, BioTechnique, 37, 517 (2004).

18. A. Pantano, Carbon Nanotube Based Composites: Processing, Properties, Modelling and Application, Smithers Rapra Technology Ltd, (2012).

19. G. Sanchez-Pomales, C. Pagan-Miranda, L. Santiago-Rodriguez, and C. R. Cabrera, in DNA-wrapped carbon nanotubes: from synthesis to application, (Ed) J. M. Marulanda, Carbon nanotubes, Ch. 35, pp.721, InTech (2010).

20. A. Jagota, B. A. Diner, S. Boussaad, and M. Zheng, in Carbon Nanotube-Biomolecule Interactions: Applications in Carbon Nanotube Separation and Biosensing, (Eds) S. V. Rotkin and S. Subramoney, Applied Physics of Carbon Nanotubes: Fundamentals of Theory, Optics and Transport Devices, Ch. 10, pp. 252, Springer, New York (2005)
21. C. Backes, in Noncovalent Functionalization of Carbon Nanotubes. Fundamental Aspects of Dispersion and Separation in Water. Ch. 1, p. 1, Springer-Verlag, Berlin (2012).

22. S. Kruss, A. J. Hilmer, J. Zhang, N. F. Reuel, B. Mu, and M. S. Strano, Adv. Drug Delivery Rev. 65, 1933 (2013).

23. P. Avouris, M. Freitag, and V. Perebeinos, in Carbon nanotube optoelectronics, (Eds.) A. Jorio, G. Dresselhaus, and M. S. Dresselhaus, Carbon Nanotubes Advanced Topics in the Synthesis, Structure, Properties and Applications. Ch. 8, p. 423, Springer, New York (2008).

24. M. S. Strano, A. A. Boghossian, W.-J. Kim, P. W. Barone, E. S. Jeng, D. A. Heller, N. Nair, J. Hong, R. Sharma, and C. Y. Lee, MRS Bulletin, 34, 950 (2009).

25. P. J. F. Harris, in Carbon nanotubes and related structures: New materials for the twenty-first century, Cambridge University Press, Cambridge, New York (2001).

26. M. S. Strano, V. C. Moore, M. K. Miller, M. J. Allen, E. H. Haroz, C. Kittrell, R. H. Hauge, and R. E. Smalley, J. Nanosci. Nanotech. 3, 81 (2003).

27. O. M. Burlaka, Y. V. Pirko, A. I. Yemets, and Y. B. Blume, in Application of carbon nanotubes for plant genetic transformation, (Eds) O. Fesenko and L. Yatsenko, Nanocomposites, nanophotonics, Nanobiotechnology, and applications, Ch. 20, p. 233, Springer, Switzerland (2015).

28. C. A. Dyke and J. M. Tour, J. Phys. Chem. A, 108, 11152 (2004).

29. J. L. Bahr, J. Yang, D. V. Kosynkin, M. J. Bronikowski, R. E- Smalley, and J. M. Tour, J.Am.Chem.Soc. 123, 6536 (2001).

30. M. Zheng, A. Jagota, E. D. Semke, A. Bruce, B. A. Diner, R. S. Mclean, S. R. Lustig, R. E. Richardson, and N. G. Tassi, Nat. Mater. 2, 338 (2003).

31. B. Alberts, D. Bray, K. Hopkin, A. Johnson, J. Lewis, M. Raff, K. Roberts, and P. Walter, Essential Cell Biology, 4 edition, Chapter 2, pp. 64, GS Garland Science Taylor and Francis Group, New York (2014).

32. A. Paul and B. Bhattacharya, Mater. Manuf. Process. 25, 891 (2010).

33. D. L. Nelson and M. M. Cox, in Principles of Biochemistry, Fifth Edition, Ch. 9, W. H. Freeman, New York (2008).

34. J. M. Berg, J. L. Tymoczko, G. J. Gatto, and L. Stryer, Biochemistry, Fifth Edition, Ch. 5, W. H. Freeman \& Company, New York (2002).

35. S. Meng and E. Kaxiras, in Interaction of DNA with CNTs: Properties and prospects for electronic sequencing. (Ed.) A. Merkoci, Biosensing using nanomaterials, Ch. 3 , p.67, WILEY, New Jersey (2009).

36. A. N. Enyashin, S. Gemming, and G. Seifert, Nanotechnology, 18, 245702 (2007).

37. S. Manohar, T. Tang, and A. Jagota, J. Phys. Chem. C, 111, 17835 (2007)

38. E. S. Jeng, A. E. Moll, A. C. Roy, J. B. Gastala, and M. S. Strano, Nano Letter, 6, 371 (2006).

39. S. C. Tsang, Z. Guo, Y. K. Chen, M. L. H. Green, H. Allen O. Hill, T. W. Hambley, and P. J. Sadler, Angewandte Chemie, 36, 2198 (1997).

40. S. Meng, P. Maragakis, C. Papaloukas, and E. Kaxiras, Nano Lett. 7, 45 (2007).

41. G. O. Gladchenko, M. V. Karachevtsev, V. S. Leontiev, V. A. Valeev, A. Y. Glamazda, A. M. Plokhotnichenko, and S. G. Stepanian, Mol. Phys., 104, 3193 (2006).

42. R. R. Johnson, A. Kohlmeyer, A. T. C. Johnson, and M. L. Klein, Nano Lett. 9, 537 (2009).

43. R. R. Johnson, A. T. C. Johnson, and M. L. Klein, Nano Lett., 8, 69 (2008).

44. W. Martin, W. Zhu, and G. Krilov, J. Phys. Chem. B, 112, 16076 (2008).

45. H. Gao and Y. Kong, Annu. Rev. Mater. Res. 34, 123 (2004).

46. G. Lu, P. Maragakis, and E. Kaxiras, Nano Lett. 5, 897 (2005)

47. M. Zheng, A. Jagota, M. S. Strano, A. P. Santos, P. Barone, S. G. Chou, B. A. Diner, M. S. Dresselhaus, R. S. Mclean, G. B. Onoa, G. G. Samsonidze, E. D. Semke, M. Usrey, and D. J. Walls, Science, 302, 1545 (2003)

48. D. A. Heller, E. S. Jeng, T. K. Yeung, B. M. Martinez, A. E. Moll, J. B. Gastala, and M. S. Strano, Science, 311, 508 (2006).

49. B. Gigliotti, B. Sakizzie, D. S. Bethune, R. M. Shelby, and J. N. Cha, Nano Lett. 6, 159 (2006).

50. S. R. Vogel, K. Müller, U. Plutowski, M. M. Kappes, and C. Richert, Phys. Stat. Sol., 244, 4026 (2007).

51. J. M. Hughes, H. Cathcart, and J. N. Coleman, J. Phys. Chem. C, 114, 11741 (2010).

52. A. Das, A. K. Sood, P. K. Maiti, M. Das, R. Varadarajan, and C. N. R. Rao, Chem. Phys. Lett., 453, 266 (2008).

53. S. Gowtham, R. H. Scheicher, R. Pandey, S. P. Karna, and R. Ahuja, Nanotechnology, 19, 125701 (2008)

54. C. A. Hunter and J. K. M. Sanders, J. Am. Chem. Soc. 112, 5525 (1990).

55. Y. Wang, J. Phys. Chem. C Nanomater Interfaces, 112, 14297 (2008)

56. Y. Zhao and D. G. Truhlar, Phys. Chem. Chem. Phys. 7, 2701 (2005)

57. M. K. Shukla, M. Dubey, E. Zakar, R. Namburu, Z. Czyznikowska, and J. Leszczynski, Chem. Phys. Lett. 480, 269 (2009).

58. J. F. Campbell, I. Tessmer, H. H. Thorp, and D. A. Erie, J. Am. Chem. Soc. 130 , 10648 (2008).

59. M. P. Landry, S. Kruss, J. T. Nelson, G. Bisker, N. M. Iverson, N. F. Reuel, and M. S. Strano, Sensors 14, 16196 (2014).

60. J. X. Wang, M. X. Li, Z. J. Shi, N. Q. Li, and Z. N. Gu, Microchem. J., 73, 325 (2002).

61. S. Malik, S. Vogel, H. Rösner, K. Arnold, F. Hennrich, A.-K. Köhler, C. Richert, and M. M. Kappes, Compos. Sci. Technol. 67, 916 (2007).

62. R. R. Johnson, A. T. C. Johnson, and M. L. Klein, Nano Lett., 8, 69 (2008).

63. G. Ao, C. Y. Khripin, and M. Zheng, J. Am. Chem. Soc., 136, 10383 (2014)

64. X. Tu, S. Manohar, A. Jagota, and M. Zheng, Nature, 460, 250 (2009).

65. D. Roxbury, A. Jagota, and J. Mittal, J Am Chem Soc. 133, 13545 (2011)

66. D. Roxbury, J. Mittal, and A. Jagota, Nano Lett., 12, 1464 (2012).

67. E. S. Jeng, J. D. Nelson, K. L. J. Prather, and M. S. Strano, Small, 6, 40 (2010). 
68. J. Zhang, A. A. Boghossian, P. W. Barone, A. Rwei, J.-H. Kim, D. Lin, D. A. Heller A. J. Hilmer, N. Nair, N. F. Reuel, and M. S. Strano, J. Am. Chem. Soc., 133, 567 (2011).

69. Z. W. Ulissi, F. Sen, X. Gong, S. Sen, N. Iverson, A. A. Boghossian, L. C. Godoy, G. N. Wogan, D. Mukhopadhyay, and M. S. Strano, Nano Lett., 14, 4887 (2014).

70. D. A. Heller, G. W. Pratt, J. Zhang, N. Nair, A. J. Hansborough, A. A. Boghossian, N. F. Reuel, P. W. Barone, and M. S. Strano, PNAS, 108, 8544 (2011).

71. S. Kruss, M. P. Landry, E. V. Ende, B. M. A. Lima, N. F. Reuel, J. Zhang, J. Nelson, B. Mu, A. Hilmer, and M. S. Strano, J. Am. Chem. Soc., 136, 713 (2014)
72. M. P. Landry, L. Vukovic, S. Kruss, G. Bisker, A. M. Landry, S. Islam, R. M. Jain, K. Schulten, and M. S. Strano, J. Phys. Chem. C, 119, 10048 (2015).

73. D. A. Heller, H. Jin, B. M. Martinez, D. Patel, B. M. Miller, T.-K. Yeung, P. V. Jena, C. Höbartner, T. Ha, S. K. Silverman, and M. S. Strano, Nature Nanotechnology, 4 , 114 (2009).

74. F. Sen, A. A. Boghossian, S. Sen, Z. W. Ulissi, J. Zhang, and M. S. Strano, ACS Nano, 6, 10632 (2012).

75. T.-G. Cha, B. A. Baker, M. D. Sauffer, J. Salgado, D. Jaroch, J. L. Rickus, D. M. Porterfield, and J. H. Choi, ACS Nano, 5, 4236 (2011) 